\section{SLIDE MAKER: A BASIC program to create 35-mm slides of verbal materials}

STEVEN L. TURNER and COLIN M. MACLEOD University of Toronto, Scarborough, Ontario, Canada

Despite the widespread use of computers to control experiments involving verbal materials, it still is necessary in many instances to present these materials on slides. This is particularly the case when an experiment is to be carried out using multiple subjects in a single session, or when a set of items includes pictures intermingled with words or sentences. Unfortunately, photographic preparation of slides can be both costly and time-consuming. As a quick and inexpensive alternative, we have developed a computerized procedure for making slides. The program, called SLIDE MAKER, is a BASIC program for the Apple II family of microcomputers (see the Appendix for a listing).

The first step in using SLIDE MAKER is to create an ASCII file of the desired verbal stimuli. Because of the flexible editing capabilities, this is best done in a word processing environment. For demonstration purposes, imagine that the materials consist of 60 single words. Each word is typed on a single line, followed by a RETURN, resulting in a column of 60 words. In the line above the first word, the number 60 is entered, to tell SLIDE MAKER the length of the file. This file is then saved to the disk containing SLIDE MAKER, and the file is named, for example, WORDS.

The listing in the Appendix presents a DOS 3.3 version of SLIDE MAKER, which accepts any DOS 3.3 text file as input. Thus, a text file created in Apple Writer would be suitable. If a ProDOS word processor is used, an ASCII file, rather than a word processor file, should be saved. Thus, with Apple Works, select the print option and print to the disk. In addition, four lines must be modified for SLIDE MAKER to run with ProDOS. In line 680, 'PR\#0' should be changed to 'PRINT CHR\$ (4); “'PR\#0"'.' Lines 5010 and 5110 should be modified similarly. In line 1210 , 'CHR\$ (13) +' should be omitted, so that the line reads 'D\$ = CHR \$ (4).'

In running SLIDE MAKER, the only constraint is that a single run can accommodate no more than 247 items, due to APPLE's limit on the length of a string array. We tend to think in terms of 240 as a maximum, because this constitutes 10 pages of letter-size paper (see below). Thus, if more than 240 items are to be created, separate text

Preparation of the program and this article was supported by Grants A7459 and E6532 from the Natural Sciences and Engineering Research Council of Canada to the second author. We thank Joseph Sidowski and an anonymous reviewer for their helpful comments on a previous version. Correspondence should be addressed to Colin M. MacLeod, Division of Life Sciences, University of Toronto, Scarborough Campus, Scarborough, Ontario M1C 1A4, Canada. files should be prepared and the program should be run once for each file.

SLIDE MAKER permits the typed output to be in either 12-pitch elite or 10-pitch pica font. To avoid touching the inner edges of a $35-\mathrm{mm}$ slide mount $(35 \mathrm{~mm}$ in width $\times$ $24 \mathrm{~mm}$ in height), the maximum number of characters per item in the text file is 15 in elite and 12 in pica. Any keyboard character can be used, in either upper- or lowercase. Thus, eligible items are single words such as "HORSE" or "mother," sets of digits such as " 973 " or " $4 \ldots 6 \ldots 2 \ldots 8$," paired associates such as "27-FROG" or "glue-CHAIR," brief sentences such as "See Spot run," warning stimuli such as "*****," and a variety of other possibilities.

Once the stimulus file has been created and saved, it is time to run SLIDE MAKER. The program requires only three inputs: the name of the relevant file (e.g., WORDS, as above), the number of lines per slide ( 1 or 2$)$, and the font $(E$ or $P$, for elite or pica). If the response to the number of lines question is 1 , a single line will appear on each slide. In the case of the example file WORDS, 60 slides will be created. If the answer is 2 , two lines will appear on each slide, one above the other. Again, in the case of the file WORDS, 30 slides will be produced, each with two printed lines. The first slide contains Word 1 above Word 2, the next Word 3 above Word 4 , and so on. It is possible to modify SLIDE MAKER to accommodate up to 8 lines per slide. Any more will not fit comfortably within the boundaries of the $35-\mathrm{mm}$ slide mount.

After the file has been loaded into memory (Subroutine 1000), SLIDE MAKER reminds the user to turn on the printer and to align the printer paper prior to starting the printout (Subroutine 5000). Setting the page at the same location used to start a word-processed document is optimal. Next, slide output begins (Subroutine 500). In the case of one line per slide (Subroutine 2000), each word in the file is printed horizontally at the center of a $38 \mathrm{~mm}$ wide $\times 35.5 \mathrm{~mm}$ high rectangle. If this rectangle is thought of as consisting of 8 lines, the word appears on line 4 from the top. The boundaries of each rectangle consist of solid and dashed lines horizontally (Subroutine 2500) and hash marks vertically (Subroutine 4000). Each letter-size page of printout contains 24 slide rectangles ( 4 columns $\times 6$ rows).

In the case of two items per slide (Subroutine 3000), the first item appears on line 3 and the second on line 5, so that they appear quite centered. SLIDE MAKER also centers each item in its rectangle by inserting appropriate spacing to the left of the item. If desired, this centering could be defeated by altering line 2040 for one-line items or Subroutine 3300 for two-line items. Page feeds are done automatically after each $4 \times 6$ matrix, and the printout stops after the last row containing an item.

A couple of features of SLIDE MAKER should be noted. The program expects to find the printer in Slot 1; 
if the user's printer is located elsewhere, line 5110 should be modified. Also, because of the centering and the maximum number of character constraints, if the printer's default mode is not 12-pitch elite or 10-pitch pica, the appropriate control sequence to switch to one of those modes should be entered in line 5115 or before running the program. Otherwise, the printed material may not fit (or be centered) in the 35-mm slide frame. A daisy-wheel printer with carbon ribbon is preferable, but a good dot matrix printer with a new ribbon will also produce acceptable quality.

When the program run is completed, the user will have a series of pages of items in rectangles. The next step is to copy these pages onto clear plastic sheets. This can be done in a single step on some photocopying machines, and produces very good quality slides. If such a photocopier is not available, the process involves two steps. The first is to make a clean, crisp photocopy of the printout page. The image of this photocopy then can be transferred to a specially treated plastic sheet by a thermal process using a Thermofax or similar machine. Using a photocopy in the thermal process results in better transfer than does using the original. With a little practice, the quality of these slides can be quite acceptable, although perhaps not as good as in the one-step procedure.

The last step is to cut out the individual rectangles and insert them into slide mounts. Two kinds of slide mounts are available at most photographic supply stores. With the first kind, plastic mounts snap together for quick assembly, although extended usage may lead to some movement of the plastic sheet within the mount. A small piece of tape that attaches the plastic sheet to one side of the mount will help to solve this problem. The second kind involves cardboard mounts that are sealed using heat. Although not quite as quick, there is no danger of slippage and these are cheaper than plastic mounts. The only drawbacks of cardboard mounts are that they can become dog-eared with extended use, and there is a risk of burning the plastic sheet during sealing if care is not taken.

We have used this program and technique successfully for several years. It is inexpensive and quick: On a number of occasions, we have produced several hundred slides in an afternoon for under $\$ 20$ (the cost of the slide mounts). It is particularly handy for such things as undergraduate projects and classroom demonstrations, but it has also been used to prepare materials for a variety of published experiments.

Although the procedure is intended for verbal materials, it can be used with other types of stimuli as well. For instance, if the file is filled with blanks followed by returns, a set of blank matrices will be generated. Using the reduce/enlarge function of a photocopier, line drawings can be created that fit within the slide rectangles. These can be glued into the rectangles, and then picture slides can be created. Indeed, the reduction function of a photocopier can even allow reduced versions of verbal stimuli longer than 15 characters to be inserted in a slide.

When slides must be used, SLIDE MAKER is an attractive alternative to slower, and much more expensive, photography. Although admittedly not photographic in quality, slides made using our procedure are very good.

Availability. The interested researcher who would like a copy of SLIDE MAKER on disk for the APPLE should send a disk and mailer to Colin M. MacLeod, Division of Life Sciences, Scarborough Campus, University of Toronto, Scarborough, ON M1C 1A4, Canada. Please indicate your preference for DOS 3.3 or ProDOS. The program will be sent by return mail. Of course, given its brevity, SLIDE MAKER is easily input from the keyboard. For those using other microcomputers, the program should be easily modifiable.

\section{APPENDIX}

Listing of SLIDE MAKER Program

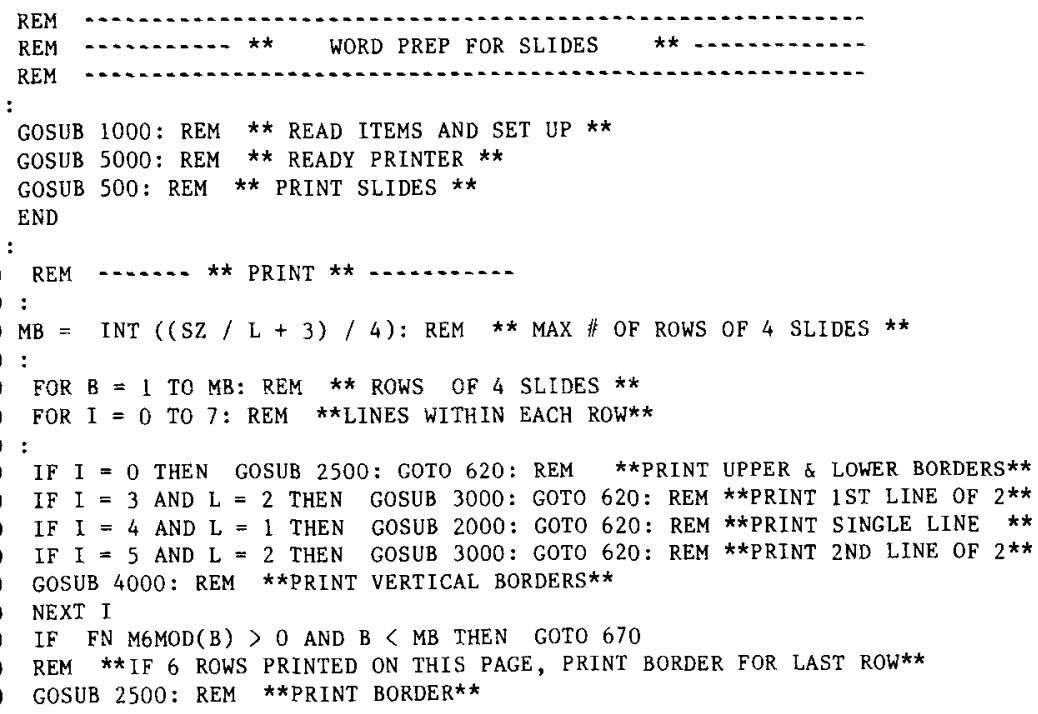




\section{APPENDIX (Continued)}

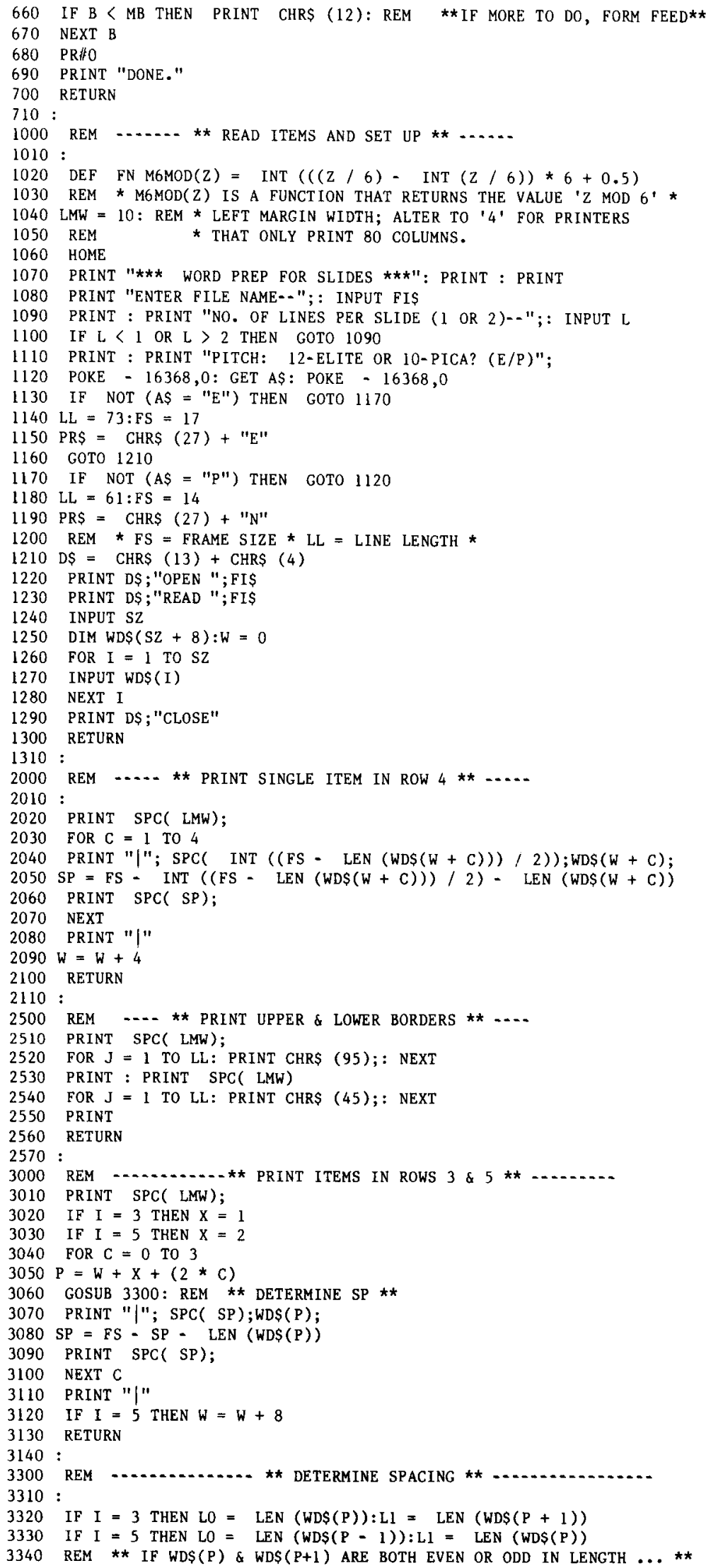




\section{APPENDIX (Continued)}

3350 IF $(($ LO $/ 2=$ INT $(L O / 2))$ AND $(L / 2=$ INT $(L / / 2)))$ OR $($ NOT $(L O / 2$ $=$ INT $($ LO $/ 2))$ AND NOT $(\mathrm{Ll} / 2=\operatorname{INT}(\mathrm{Ll} / 2)))$ THEN SP $=$ INT ((FS - LEN (WDS(P))) / 2): GOTO 3380

3360 IF $I=3$ THEN GOSUB 3500

3370 IF $I=5$ THEN GOSUB 3800

3380 RETURN

3390 :

3500 REM $\ldots \ldots \ldots \ldots \ldots \ldots$.

$3510:$

3520 REM * WHEN FS \& LEN WDS(P) BOTH EVEN OR ODD, NO CORRECTION

3530 IF ((FS / $2=$ INT (FS / 2)) AND (LO / $2=$ INT (LO / 2))) OR ( NOT (FS / 2

$=\operatorname{INT}($ FS $/ 2)$ ) AND NOT (LO / $2=\operatorname{INT}($ LO $/ 2))$ ) THEN GOTO 3560

3540 IF LO $>$ LI THEN LO $=$ LO -1

3550 IF LO $<$ LI THEN LO $=\mathrm{L} 0+1$

$3560 \mathrm{SP}=(\mathrm{FS}-\mathrm{LO}) / 2$

3570 RETURN

$3580:$

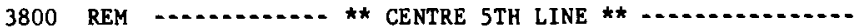

3810 :

3820 REM * WHEN FS \& LEN WD\$(P) BOTH EVEN OR ODD, NO CORRECTION *

3830 IF ((FS / 2 = INT (FS / 2)) AND (Ll / 2 = INT $(\mathrm{Ll} / 2))$ ) OR ( NOT (FS / 2

$=\operatorname{INT}($ FS $/ 2)$ ) AND NOT (L1 / 2 = INT (L1 / 2))) THEN GOTO 3860

3840 IF L1 $>$ LO THEN L1 $=\mathrm{Ll}-1$

3850 IF $\mathrm{Ll}<\mathrm{L} 0$ THEN $\mathrm{Ll}=\mathrm{L} 1+1$

$3860 \mathrm{SP}=(\mathrm{FS}-\mathrm{L} 1) / 2$

3870 RETURN

3880 :

4000 REM $\ldots . . \star \star$ PRINT APPROPRIATE VERTICALS $* \star \ldots .$.

4010:

4020 PRINT SPC( LMW);

4030 PRINT "|"; SPC( FS);"|"; $\operatorname{SPC}($ FS );"|"; $\operatorname{SPC}($ FS $) ; " \mid " ; \operatorname{SPC}($ FS );"|"

4040 RETURN

4050 :

5000 REM $\ldots . . . * *$ ALIGN PRINTER PAPER $* \star \ldots \ldots$

5010 PR: 0

5020 HOME : PRINT CHR\$ (7); CHR\$ (7)

5030 FLASH

5040 PRINT "ATTENTION": NORMAL : PRINT : PRINT

5050 PRINT "PLEASE TURN ON PRINTER."

5060 PRINT : PRINT "PLEASE ALIGN PRINTER PAPER T0"

5070 PRINT : PRINT "BEGIN PRINTING."

5080 PRINT : PRINT "PRESS ANY KEY WHEN READY."

5090 POKE - 16368,0: GET AS

5100 HOME

5110 PR\#1

5115 PRINT PRS;: REM * SET PRINTER PITCH *

5120 PRINT CHRS (9);"90N"

5130 RETURN

5140 :

(Revision accepted for publication February 23, 1988.) 